\title{
Spectral Functions for the Tomonaga-Luttinger and Luther-Emery Liquids
}

\author{
Dror Orgad \\ Department of Physics, University of California, Los Angeles, CA 90095-1547
}

(November 6, 2018)

\begin{abstract}
We calculate the finite temperature single hole spectral function and the spin dynamic structure factor of spinfull one-dimensional Tomonaga-Luttinger liquid. Analytical expressions are obtained for a number of special cases. We also calculate the single hole spectral function of a spin gapped Luther-Emery liquid and obtain exact results at the free fermion point $K_{s}=1 / 2$. These results may be applied to the analysis of angle resolved photoemission and neutron scattering experiments on quasi-one-dimensional materials.
\end{abstract}

\section{INTRODUCTION}

The interacting one-dimensional electron gas (1DEG) has intrighed physicists since the pioneering work of Tomonagal. Through the collective effort of many of them, and especially due to the advancement of the bosonization technique日 4 , a large number of its properties have been discovered.

The past two decades have witnessed an experimental effort to identify and study, using various probes, quasione-dimensional systems. Among the materials studied one finds organic compounds such as polyacetyleneand the Bechgaard salts 9 as well as severat families of inorganic quasi-one-dimensional materials 4 (for example, the transition metal bronzes). The edge states in the quantum Hall effect constitute yet another realization of one-dimensional interacting systems. Progress made in nanoscale fabrication techniques have made it possible to artificially define one-dimensional channels in two-dimensional electronic heterostructure 10 . Most recently, experimental evidence has emerged for the existence of stripe phases in doped antiferromagnets, such as the copper-oxide high temperature superconductors 11 . In these phases the doped holes segregate into quasione-dimensional metallic regions embedded in a predominantly antifferomagnetic background.

In recent years angle resolved photoemission spectroscopy (ARPES) has matured into a powerful experimental method for probing the single particle properties of strongly correlated systems. ARPES measurements on the Bechgaard salts 12 , the transition metalchalcogenide 13 , the blue bronzes 14 and the cuprates 15 have provided evidence in favor of correlated one-dimensional physics although full agreement with the predicted theoretical picture is still lacking.

The zero temperature correlation functions of the gapless Tomonaga-Luttinger liquid, and in particular the single hole spectral function which is measured by ARPES, have been calculated previously for the spinless 1 as well as for the spinfull case 6107 . Extending these results to finite temperatures is desirable on the following grounds. First, real experiments are carried out at finite temper- atures and should be contrasted with theoretical predictions relevant for such conditions. Secondly, the Tomonaga-Luttinger liquid is a quantum critical system which consequently exhibits scaling behavior. It is a rare example where not only the scaling exponents but indeed the entire scaling functions can be computed explicitly. In Section II we obtain the scaling form of the single hole spectral function and the spin dynamic structure factor of the spinfull Tomonaga-Luttinger liquid. Closed form analytical expressions are derived for a number of special cases. These results complement previous numerical evaluations of the finite temperature spectral functions 18.19 and allow for easy investigation of their properties in few limits which are of physical interest. Integrals of these spectral functions are considered as well.

When backward scattering of electrons becomes relevant the 1DEG develops a-pin gap and is described by the Luther-Emery liquidE0 (Umklapp processes may create a charge gap). While the spectrum of the LutherEmery liquid can be readily derived at the special "free fermion" point $K_{s}=1 / 2$, the evaluation of electronic correlation functions there is non-trivial due to their highly non-local form in terms of the pseudo- fermions. Several authors have made progress in this direction, see Refs. 21.22, and in section III we extend and correct their study and obtain exact expressions for the single hole spectral function at the free fermion point for temperatures much smaller than the spin gap. Some technical details are relegated to appendices.

\section{THE TOMONAGA-LUTTINGER LIQUID}

\section{A. The model}

The Tomonaga-Luttinger model embodies the low energy and long wavelength physics of the 1DEG. It is composed of two branches of left $(\eta=-1)$ and right $(\eta=+1)$ moving massless Dirac fermions constructed around the left and right Fermi points of the 1DEG. We will consider the case where the fermions carry spin $1 / 2$ and denote by $\sigma= \pm 1$ their spin polarization. In the absence of back- 
ward scattering and Umklapp processes the Hamiltonian density is given by $(\hbar=1)$

$$
\begin{aligned}
\mathcal{H}= & -v_{F} \sum_{\eta, \sigma= \pm 1} \eta \psi_{\eta, \sigma}^{\dagger} i \partial_{x} \psi_{\eta, \sigma} \\
& +\frac{1}{2} \sum_{\eta= \pm 1} g_{2, c} \rho_{\eta}(x) \rho_{-\eta}(x)+g_{4, c} \rho_{\eta}(x) \rho_{\eta}(x) \\
& +2 \sum_{\eta= \pm 1} g_{2, s} \mathrm{~S}_{\eta}^{z}(x) \mathrm{S}_{-\eta}^{z}(x)+g_{4, s} \mathrm{~S}_{\eta}^{z}(x) \mathrm{S}_{\eta}^{z}(x)
\end{aligned}
$$

where $v_{F}$ is the noninteracting Fermi velocity and

$$
\begin{aligned}
\rho_{\eta} & =\sum_{\sigma} \psi_{\eta, \sigma}^{\dagger} \psi_{\eta, \sigma}, \\
\mathbf{S}_{\eta} & =\frac{1}{2} \sum_{\sigma, \sigma^{\prime}} \psi_{\eta, \sigma}^{\dagger} \boldsymbol{\tau}_{\sigma \sigma^{\prime}} \psi_{\eta, \sigma^{\prime}},
\end{aligned}
$$

with $\tau$ being the Pauli matrices. Despite its appearance, the last term in (11) does not break the $S U(2)$ spin symmetry, since $\mathrm{S}_{\eta}^{z^{2}}(x)=\lim _{x^{\prime} \rightarrow x} \mathbf{S}_{\eta}(x) \cdot \mathbf{S}_{\eta}\left(x^{\prime}\right) / 2+\rho_{\eta}^{2}(x) / 8$; the $g_{2, s}$ term is the only term which breaks this symmetry.

In order to compute the single particle preperties of the model one uses the bosonization identity 2324

$$
\psi_{\eta, \sigma}=\frac{1}{\sqrt{2 \pi a}} F_{\eta, \sigma} \exp \left[i \eta k_{F} x-i \Phi_{\eta, \sigma}(x)\right],
$$

which expresses the fermionic fields in terms of selfdual fields $\Phi_{\eta, \sigma}(x)$ obeying $\left[\Phi_{\eta, \sigma}(x), \Phi_{\eta^{\prime}, \sigma^{\prime}}\left(x^{\prime}\right)\right]=$ $-i \pi \delta_{\eta, \eta^{\prime}} \delta_{\sigma, \sigma^{\prime}} \operatorname{sign}\left(x-x^{\prime}\right)$. The Klein factors $F_{\eta, \sigma}$ are responsible for reproducing the correct anti-commutation relations between different fermionic species and $a$ is a short distance cutoff that is taken to zero at the end of the calculation. Here and throughout the paper we consider the limit where the size of the system $L$ is taken to infinity and correspondingly ignore terms of the order $1 / L$ (for a discussion of finite size effects in the finite temperature case see Refs. 2526).

The demonstration of the celebrated charge-spin separation in the model is facilitated by expressing $\Phi_{\eta, \sigma}$ in terms of the bosonic fields $\phi_{c}, \phi_{s}$ and their conjugated momenta $\partial_{x} \theta_{c}, \partial_{x} \theta_{s}$

$$
\Phi_{\eta, \sigma}=\sqrt{\pi / 2}\left[\left(\theta_{c}-\eta \phi_{c}\right)+\sigma\left(\theta_{s}-\eta \phi_{s}\right)\right],
$$

in terms of which the charge and spin densities are given by

$$
\begin{gathered}
\rho(x)=\sum_{\eta} \rho_{\eta}(x)=\sqrt{2 / \pi} \partial_{x} \phi_{c}, \\
\mathrm{~S}^{z}(x)=\sum_{\eta} \mathrm{S}_{\eta}^{z}(x)=\sqrt{1 / 2 \pi} \partial_{x} \phi_{s} .
\end{gathered}
$$

The decomposition (5) also facilitates the diagonalization of the Hamiltonian. It becomes a sum of two independent pieces describing noninteracting charge and spin density waves which are the elementary excitations of the system

$$
\mathcal{H}=\sum_{\alpha=c, s} \frac{v_{\alpha}}{2}\left[K_{\alpha}\left(\partial_{x} \theta_{\alpha}\right)^{2}+\frac{\left(\partial_{x} \phi_{\alpha}\right)^{2}}{K_{\alpha}}\right]
$$

The velocities of the collective modes are

$$
v_{\alpha}=\sqrt{\left(v_{F}+\frac{g_{4, \alpha}}{\pi}\right)^{2}-\left(\frac{g_{2, \alpha}}{\pi}\right)^{2}},
$$

and the parameters $K_{\alpha}$, which determine the power-law behavior of the correlation functions, read

$$
K_{\alpha}=\sqrt{\frac{\pi v_{F}+g_{4, \alpha}-g_{2, \alpha}}{\pi v_{F}+g_{4, \alpha}+g_{2, \alpha}}} .
$$

The effective parameters that enter the Hamiltonian depend on the specifics of the model for which (11) is a low energy fixed point. We note, however, that spin-rotation invariance dictates $g_{2, s}=0$ and consequently $K_{s}=1$.

\section{B. The space-time correlation functions}

The bosonized expression for the fermion field operators (4) and the fact that the theory reduces to that of free bosons make it straightforward to derive expressions for the space-time response functions (which we denote with a tilde). We will focus on the finite temperature single hole Green function

$$
\tilde{G}_{\eta}^{<}(x, t ; T)=\left\langle\psi_{\eta, \sigma}^{\dagger}(x, t) \psi_{\eta, \sigma}(0,0)\right\rangle .
$$

We compute this Green function, rather than the more usual time ordered or retarded Green functions, because it includes only the one-hole states. This fact makes it relevant to ARPES whose cross section is directly proportional to the Fourier transform of $\tilde{G}^{<}$. Other Green functions can be easily obtained from it, since for the model (11) $\tilde{G}_{\eta}^{>}(x, t ; T)=\left\langle\psi_{\eta, \sigma}(0,0) \psi_{\eta, \sigma}^{\dagger}(x, t)\right\rangle=\tilde{G}_{\eta}^{<}(-x,-t ; T)$.

We will also consider the $2 k_{F}$ component of the transverse spin dynamic structure factor, which is measured by polarized neutron scattering

$$
\begin{aligned}
\tilde{\mathcal{S}}(x, t ; T) & =\left\langle\mathrm{S}_{2 k_{F}}^{x}{ }^{\dagger}(x, t) \mathrm{S}_{2 k_{F}}^{x}(0,0)\right\rangle \\
& +\left\langle\mathrm{S}_{2 k_{F}}^{y}{ }^{\dagger}(x, t) \mathrm{S}_{2 k_{F}}^{y}(0,0)\right\rangle .
\end{aligned}
$$

where

$$
\mathbf{S}_{2 k_{F}}=\frac{1}{2} \sum_{\sigma, \sigma^{\prime}} \psi_{1, \sigma}^{\dagger} \boldsymbol{\tau}_{\sigma \sigma^{\prime}} \psi_{-1, \sigma^{\prime}}
$$

and the $\boldsymbol{\tau}$ are the Pauli matrices.

The Tomonaga-Luttinger liquid (1]) is a quantum critical system. It also exhibits spin-charge separation. This implies a scaling form for the response functions with separate spin and charge pieces. Specifically one finds 23 


$$
\begin{aligned}
\tilde{G}_{\eta}^{<}(x, t ; T)= & \frac{1}{2 \pi a} e^{-i \eta k_{F} x}\left(\frac{a}{\lambda_{T, c}}\right)^{2 \gamma_{c}+\frac{1}{2}}\left(\frac{a}{\lambda_{T, s}}\right)^{2 \gamma_{s}+\frac{1}{2}} \\
& \times \tilde{g}_{c}\left(\frac{x}{\lambda_{T, c}}, \frac{v_{c} t}{\lambda_{T, c}}\right) \tilde{g}_{s}\left(\frac{x}{\lambda_{T, s}}, \frac{v_{s} t}{\lambda_{T, s}}\right), \quad(14)
\end{aligned}
$$

where we introduced the thermal lengths $\left(k_{B}=1\right)$

$$
\lambda_{T, \alpha}=\frac{v_{\alpha}}{\pi T},
$$

and the exponents

$$
\gamma_{\alpha}=\frac{1}{8}\left(K_{\alpha}+K_{\alpha}^{-1}-2\right)
$$

defined so that $\gamma_{\alpha}=0$ for noninteracting fermions. Since the spin and charge sectors are formally invariant under separate Lorentz transformations the functions $\tilde{g}_{\alpha}$ also spilt into right and left moving parts

$$
\tilde{g}_{\alpha}(x, t)=\tilde{h}_{\gamma_{\alpha}+\frac{1}{2}}(\eta x-t) \tilde{h}_{\gamma_{\alpha}}^{*}(\eta x+t),
$$

where

$$
\tilde{h}_{\gamma}(x)=[-i \sinh (x+i a)]^{-\gamma} .
$$

Similarly, for the spin correlation function one finds 23

$$
\begin{aligned}
\tilde{\mathcal{S}}(x, t ; T)= & \frac{1}{(2 \pi a)^{2}} e^{-2 i k_{F} x}\left(\frac{a}{\lambda_{T, c}}\right)^{2 \beta_{c}}\left(\frac{a}{\lambda_{T, s}}\right)^{2 \beta_{s}} \\
& \times \tilde{C}_{c}\left(\frac{x}{\lambda_{T, c}}, \frac{v_{c} t}{\lambda_{T, c}}\right) \tilde{C}_{s}\left(\frac{x}{\lambda_{T, s}}, \frac{v_{s} t}{\lambda_{T, s}}\right),
\end{aligned}
$$

where

$$
\tilde{C}_{\alpha}(x, t)=\tilde{h}_{\beta_{\alpha}}(x-t) \tilde{h}_{\beta_{\alpha}}^{*}(x+t) .
$$

Here we introduced the exponents

$$
\beta_{c}=\frac{K_{c}}{2} \quad, \quad \beta_{s}=\frac{1}{2 K_{s}} .
$$

We note that the perpendicular component of the spin dynamic structure factor

$$
\tilde{\mathcal{S}}^{z}(x, t ; T)=\left\langle\mathrm{S}_{2 k_{F}}^{z}{ }^{\dagger}(x, t) \mathrm{S}_{2 k_{F}}^{z}(0,0)\right\rangle,
$$

is obtained from the result for the transverse part (19) after multiplying it by an overall factor of $1 / 2$ and using the exponents

$$
\beta_{c}=\frac{K_{c}}{2} \quad, \quad \beta_{s}=\frac{K_{s}}{2},
$$

instead of (21). This holds true also for the spectral functions calculated in the following subsections. Of coarse, in the spin rotation invariant case $\tilde{\mathcal{S}}^{z}=\tilde{\mathcal{S}} / 2$.

\section{The spectral functions}

It is a general feature of the field-theoretic approach to this problem that relatively simple expressions are obtained for the space-time dependent correlation functions. For comparison with experiments, however, we are typically interested in the Fourier transform of these correlation functions. Conceptually, this is simple, and indeed, to evaluate the Fourier transform, we need only to perform a two dimensional integral. However, it is not generally simple to carry out this calculation analytically. Below we consider the cases where this can be done.

As we noted before, the critical nature of the model leads to a scaling form for the spectral functions. A simplifying feature introduced by the spin-charge separation is the ability to express these scaling functions as a convolution of spin and charge parts

$$
\begin{aligned}
G^{<}(\tilde{k}, \tilde{\omega} ; T) & =\int_{-\infty}^{\infty} d x d t e^{i\left[\eta\left(k+k_{F}\right) x-\omega t\right]} \tilde{G}_{\eta}^{<}(x, t ; T) \\
& =\frac{a}{(2 \pi)^{3} v_{c}}\left(\frac{a}{\lambda_{T, c}}\right)^{2 \gamma_{c}-\frac{1}{2}}\left(\frac{a}{\lambda_{T, s}}\right)^{2 \gamma_{s}-\frac{1}{2}} \\
& \times \int_{-\infty}^{\infty} d q d \nu g_{c}(q, \nu) g_{s}(\tilde{k}-r q, \tilde{\omega}-\nu)
\end{aligned}
$$

where we introduce the velocity ratio $r=v_{s} / v_{c}$, define the scaling variables

$$
\tilde{k}=\frac{v_{s} k}{\pi T} \quad, \quad \tilde{\omega}=\frac{\omega}{\pi T},
$$

and

$$
g_{\alpha}(k, \omega)=\frac{1}{2} h_{\gamma_{\alpha}+\frac{1}{2}}\left(\frac{\omega+k}{2}\right) h_{\gamma_{\alpha}}\left(\frac{\omega-k}{2}\right) .
$$

$h_{\gamma}(k)$, the Fourier transform of $\tilde{h}_{\gamma}(x)$, is evaluated in Appendix A, where some of its properties are listed as well. For non-integer values of $\gamma$ it is given by

$$
h_{\gamma}(k)=\operatorname{Re}\left[(2 i)^{\gamma} B\left(\frac{\gamma-i k}{2}, 1-\gamma\right)\right],
$$

where $B(x, y)$ is the Beta function.

In a similar fashion we obtain for the spin susceptibility

$$
\begin{aligned}
\mathcal{S}(\tilde{k}, \tilde{\omega} ; T) & =\int_{-\infty}^{\infty} d x d t e^{i\left[\left(k+2 k_{F}\right) x-\omega t\right]} \tilde{\mathcal{S}}(x, t ; T) \\
& =\frac{1}{(2 \pi)^{4} v_{c}}\left(\frac{a}{\lambda_{T, c}}\right)^{2 \beta_{c}-1}\left(\frac{a}{\lambda_{T, s}}\right)^{2 \beta_{s}-1} \\
& \times \int_{-\infty}^{\infty} d q d \nu C_{c}(q, \nu) C_{s}(\tilde{k}-r q, \tilde{\omega}-\nu)
\end{aligned}
$$

where 


$$
C_{\alpha}(k, \omega)=\frac{1}{2} h_{\beta_{\alpha}}\left(\frac{\omega+k}{2}\right) h_{\beta \alpha}\left(\frac{\omega-k}{2}\right) .
$$

Henceforth $k$ is measured relative to $k_{F}$ and $2 k_{F}$ when computing $G^{<}$and $\mathcal{S}$, respectively. We also define the Fourier transform with respect to $\eta x$. This has the effect of making $k$ positive outside the Fermi surface and negative inside; thus giving the same expression for the spectral function of left and right moving holes.

Here we would like to note the non-commutativity of the limits $k, \omega \rightarrow 0$ and $T \rightarrow 0$ in calculating the zero temperature dc response of the system. Due to the scaling form of the spectral functions taking the zero temperature limit first gives a result which is determined by the $\tilde{k}, \tilde{\omega} \rightarrow \infty$ behavior of the scaling functions, while it is the $\tilde{k}, \tilde{\omega} \rightarrow 0$ behavior which is relevant in case $k$ or $\omega$ is set to zero from the outset.

Further analytic progress in calculating the spectral functions can be achieved in the following cases:

\section{The case $v_{c}=v_{s}$}

Since there is no symmetry between the charge and spin sectors, we generally expect that the spin and charge velocities are different. This greatly complicates the explicit calculation of the Fourier transforms, as the model is not "Lorentz invariant" under transformations that involve both the spin and charge sectors. Such an invariance is restored if $v_{c}=v_{s}=v$. In this case the correlation functions have the same form as those of a related model of spinless electrons, for which the zero temperature spectral functions have been calculated by Luther and Peschellt.

The major simplification that follows directly from the fact that the charge and spin velocities are equal is that the two-dimensional Fourier transform reduces to a product of two one-dimensional transforms - one for the right moving piece and one for the left moving piece. Specifically one finds, with $\gamma_{G}=\gamma_{c}+\gamma_{s}$, that

$$
\begin{aligned}
G^{<}(\tilde{k}, \tilde{\omega} ; T) & =\frac{1}{4 \pi^{2} T}\left(\frac{a}{\lambda_{T}}\right)^{2 \gamma_{G}} \\
& \times h_{\gamma_{G}+1}\left(\frac{\tilde{\omega}+\tilde{k}}{2}\right) h_{\gamma_{G}}\left(\frac{\tilde{\omega}-\tilde{k}}{2}\right) .
\end{aligned}
$$

A similar analytic form can be obtained for the spin correlation function

$$
\begin{aligned}
\mathcal{S}(\tilde{k}, \tilde{\omega} ; T) & =\frac{1}{8 \pi^{2} v}\left(\frac{a}{\lambda_{T}}\right)^{2\left(\beta_{\mathcal{S}}-1\right)} \\
& \times h_{\beta_{\mathcal{S}}}\left(\frac{\tilde{\omega}+\tilde{k}}{2}\right) h_{\beta_{\mathcal{S}}}\left(\frac{\tilde{\omega}-\tilde{k}}{2}\right),
\end{aligned}
$$

where $\beta_{\mathcal{S}}=\beta_{c}+\beta_{s}$.

\section{The spin-rotationally invariant case $\left(K_{s}=1\right)$}

We already noted that when the system is invariant under spin rotations $K_{s}=1\left(\gamma_{s}=0\right)$. At this important special point there is no mixing between left and right moving spin excitations. As a result the expression for the hole spectral function is simplified, due to the appearance of the factor $h_{0}$ in the spin part of (26), and can be expressed as a single integral

$$
\begin{aligned}
& G^{<}(\tilde{k}, \tilde{\omega} ; T)=\frac{r^{1 / 2}}{4 \pi^{3} T}\left(\frac{a}{\lambda_{T, c}}\right)^{2 \gamma_{c}} \int_{-\infty}^{\infty} d q h_{\frac{1}{2}}(\tilde{k}-2 r q) \\
& \times h_{\gamma_{c}+\frac{1}{2}}\left[\frac{\tilde{\omega}-\tilde{k}}{2}+(1+r) q\right] h_{\gamma_{c}}\left[\frac{\tilde{\omega}-\tilde{k}}{2}-(1-r) q\right] .
\end{aligned}
$$

When $K_{c}=1$ as well, the integral in (32) is trivial and we obtain

$$
G^{<}(\tilde{k}, \tilde{\omega} ; T)=\frac{1}{2 \pi^{2} T} \frac{r^{1 / 2}}{|1-r|} h_{\frac{1}{2}}\left(\frac{\tilde{\omega}-\tilde{k}}{1-r}\right) h_{\frac{1}{2}}\left(\frac{r \tilde{\omega}-\tilde{k}}{r-1}\right) .
$$
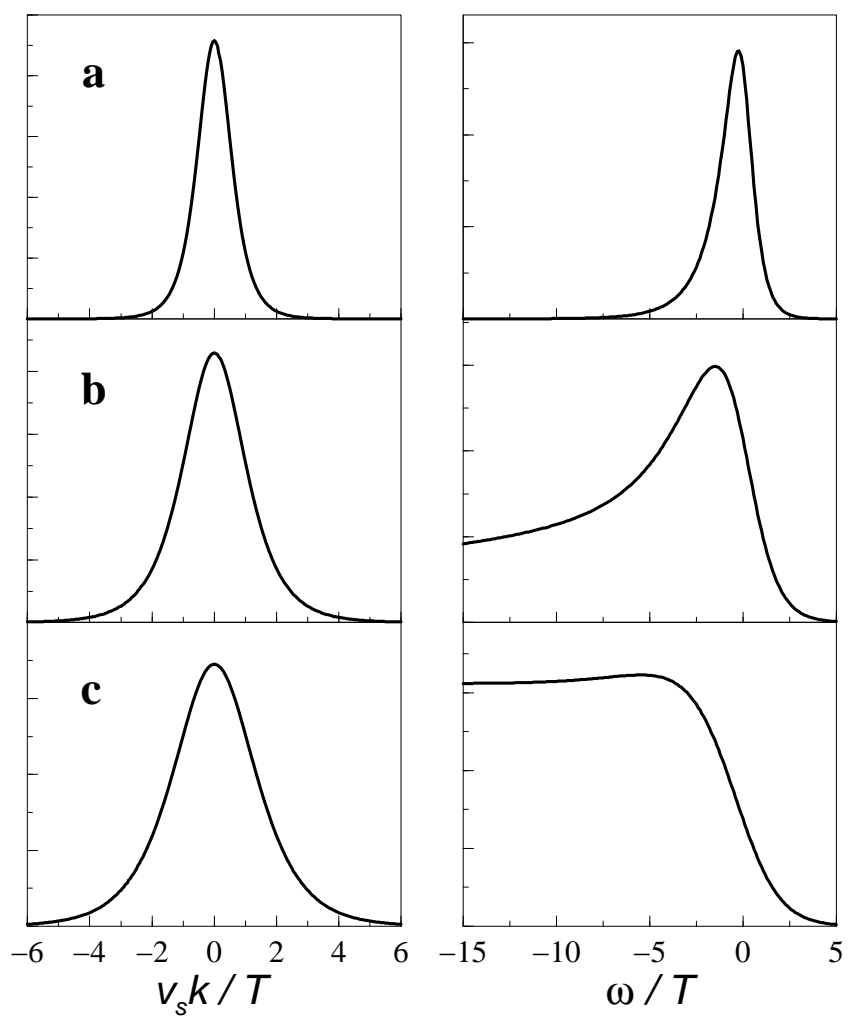

FIG. 1. MDCs at $\omega=0$ (left) and EDCs at $k=0$ (right), for a spin rotationally invariant Tomonaga-Luttinger liquid, plotted versus $v_{s} k / T$ and $\omega / T$ respectively, with $v_{c} / v_{s}=3$ and a) $\gamma_{c}=0$, b) $\gamma_{c}=0.25$, and c) $\gamma_{c}=0.5$. 
The case $\gamma_{s}=\gamma_{c}=0$ is unique in the sense that there is no mixing between left and right moving excitations. As a result there are severe kinematic constraints on $G^{<}$ that make it non-vanishing, in the zero temperature limit, only in a wedge in $k-\omega$ plane defined by the lines $\omega=v_{s} k$ and $\omega=v_{c} k$ for $k<0$. While $K_{s}=1$ reflects a symmetry of the problem it is unlikely that $K_{c}=1\left(\gamma_{c}=0\right)$ is realized in any interacting system. However, if the effective interactions are not too strong then $\gamma_{c}$ may be small. For instance, for the Hubbard model27, even in the $U / t \rightarrow \infty$ limit, $\gamma_{c}=1 / 16$. For such systems we expect (33) to be qualitatively correct with the exception of the behavior of $G^{<}$outside the above mentioned wedge. In contrast to the result for $\gamma_{c}=0$ the $T=0$ support of $G^{<}$, for non-zero $\gamma_{c}$, extends up to the line $\omega=-v_{c} k$ with $k>0$. Nevertheless, if the mixing between left and right moving charge excitations (i.e $\gamma_{c}$ ) is small the amplitude of $G^{<}$ outside the wedge is small and its gross features resemble the $\gamma_{c}=0$ result.

The customary way to present ARPES data is by plotting momentum distribution curves (MDCs) and energy distribution curves (EDCs). These curves are cuts in $G^{<}(k, \omega)$ of constant $\omega$ and constant $k$ respectively. In Fig. 1 we present MDCs at the Fermi energy $(\omega=0)$ and EDCs at the Fermi wave-vector $(k=0)$ for a spin rotationally invariant Tomonaga-Luttinger liquid for various values of the parameter $\gamma_{c}$.

\section{The case $v_{s} / v_{c} \rightarrow 0$}

In the limit where one of the velocities is much smaller than the other the calculation becomes again more tractable. In many physical systems $v_{c}>v_{s}$ and we will consider this case (for example for the $t-J$ model away from half filling we have $v_{s} / v_{c} \sim J / t<1$ ). In the limit $r=v_{s} / v_{c} \rightarrow 0$ the spin piece in Eq. (24) becomes $q$-independent and any singularities in $G^{<}$disperse with the slow velocity $v_{s}$. The $q$ integral is then readily evaluated by expressing the factors in $g_{c}$ as Fourier transforms of their real-space counterparts. The result is

$$
\begin{aligned}
& G^{<}(\tilde{k}, \tilde{\omega} ; T)=\frac{a}{(2 \pi)^{2} v_{c}}\left(\frac{a}{\lambda_{T, c}}\right)^{2 \gamma_{c}-\frac{1}{2}}\left(\frac{a}{\lambda_{T, s}}\right)^{2 \gamma_{s}-\frac{1}{2}} \\
& \quad \times \int_{-\infty}^{\infty} d \nu h_{2 \gamma_{c}+\frac{1}{2}}(\tilde{\omega}-\tilde{k}-2 \nu) h_{\gamma_{s}+\frac{1}{2}}(\tilde{k}+\nu) h_{\gamma_{s}}(\nu) .
\end{aligned}
$$

A similar calculation gives for the spin spectral function

$$
\begin{aligned}
& \mathcal{S}(\tilde{k}, \tilde{\omega} ; T)=\frac{1}{(2 \pi)^{3} v_{c}}\left(\frac{a}{\lambda_{T, c}}\right)^{2 \beta_{c}-1}\left(\frac{a}{\lambda_{T, s}}\right)^{2 \beta_{s}-1} \\
& \quad \times \int_{-\infty}^{\infty} d \nu h_{2 \beta_{c}}(\tilde{\omega}-\tilde{k}-2 \nu) h_{\beta_{s}}(\tilde{k}+\nu) h_{\beta_{s}}(\nu) .
\end{aligned}
$$

At the spin-rotationally invariant point $\left(K_{s}=1\right)$ the integral in (34) can be performed to give a result for $G^{<}$ in the limit $v_{s} / v_{c} \rightarrow 0$ but arbitrary $\gamma_{c}$

$$
G^{<}(\tilde{k}, \tilde{\omega} ; T)=\frac{r^{1 / 2}}{2 \pi^{2} T}\left(\frac{a}{\lambda_{T, c}}\right)^{2 \gamma_{c}} h_{2 \gamma_{c}+\frac{1}{2}}(\tilde{\omega}-\tilde{k}) h_{\frac{1}{2}}(\tilde{k}) .
$$

\section{Integrals of the spectral functions}

Integrals of the spectral functions, such as the density of states and the momentum occupation number, are of interest too. These simpler quantities give us a qualitative view of the spectrum, without too many complicated details.

$$
\text { 1. } \rho^{<}(\omega) \text { and } \mathcal{S}_{0}(\omega)
$$

The calculation of the density of states $\rho^{<}(\omega)$ and its analogous quantity for the spin susceptibilities $\mathcal{S}_{0}(\omega)$ is straightforward. The results are

$$
\begin{array}{r}
\rho^{<}(\tilde{\omega} ; T)=\int_{-\infty}^{\infty} \frac{d t}{2 \pi} e^{i \omega t} \tilde{G}_{\eta}^{<}(0, t)=\int_{-\infty}^{\infty} d k G^{<}(k,-\omega) \\
=\frac{1}{4 \pi^{2}} \frac{1}{\sqrt{v_{c} v_{s}}}\left(\frac{a}{\lambda_{T, c}}\right)^{2 \gamma_{c}}\left(\frac{a}{\lambda_{T, s}}\right)^{2 \gamma_{s}} h_{2 \gamma_{G}+1}(-\tilde{\omega})
\end{array}
$$

$$
\begin{aligned}
& \mathcal{S}_{0}(\tilde{\omega} ; T)=\int_{-\infty}^{\infty} \frac{d t}{2 \pi} e^{i \omega t} \tilde{\mathcal{S}}(0, t)=\int_{-\infty}^{\infty} d k \mathcal{S}(k,-\omega) \\
& =\frac{1}{8 \pi^{3} a} \frac{1}{\sqrt{v_{c} v_{s}}}\left(\frac{a}{\lambda_{T, c}}\right)^{2 \beta_{c}-\frac{1}{2}}\left(\frac{a}{\lambda_{T, s}}\right)^{2 \beta_{s}-\frac{1}{2}} h_{2 \beta_{\mathcal{S}}}(-\tilde{\omega}) .
\end{aligned}
$$

$$
\text { 2. } n(k) \text { and } S(k) \text { for the case } v_{c}=v_{s}
$$

The evaluation of the momentum occupation number $n(k)$ and the spin structure factor $S(k)$ is complicated by the fact that they can not be expressed in terms of the functions $h_{\gamma}(k)$. Contrary to the scaling functions considered above the scaling functions of these quantities depend on the cut-off $a^{-1}$. It is, however, possible to compute them in the case where the charge and spin velocities are equal.

$$
\begin{aligned}
n(\tilde{k} ; T) & =\int_{-\infty}^{\infty} d x e^{i \eta\left(k+k_{F}\right) x} \tilde{G}_{\eta}^{<}(x, 0)=\int_{-\infty}^{\infty} \frac{d \omega}{2 \pi} G^{<}(k, \omega) \\
& =-\frac{2^{\gamma_{G}+1}}{\pi}\left(\frac{a}{\lambda_{T}}\right)^{2 \gamma_{G}} \\
& \times \operatorname{Im}\left\{\int_{0}^{\infty} d x \frac{e^{i \tilde{k} x} \sinh \left(x-i a / \lambda_{T}\right)}{\left[\cosh (2 x)-\cosh \left(2 i a / \lambda_{T}\right)\right]^{\gamma_{G}+1}}\right\},
\end{aligned}
$$


where again $\gamma_{G}=\gamma_{c}+\gamma_{s}$. Since the integrand in (39) is imaginary in the interval $\left[i a / \lambda_{T}, 0\right]$ of the imaginary axis, we can add the integral along this interval to the one already present in (39) without affecting $n(k)$.

$$
\begin{aligned}
& n(\tilde{k} ; T)=\frac{2^{\gamma_{G}-1}}{\pi}\left(\frac{a}{\lambda_{T}}\right)^{2 \gamma_{G}} \\
& \times \operatorname{Im}\left\{\int_{2 i a / \lambda_{T}}^{\infty} d x \frac{e^{\left(i \frac{\tilde{k}}{2}-\frac{1}{2}\right) x+i a / \lambda_{T}}-e^{\left(i \frac{\tilde{k}}{2}+\frac{1}{2}\right) x-i a / \lambda_{T}}}{\left[\cosh (x)-\cosh \left(2 i a / \lambda_{T}\right)\right]^{\gamma_{G}+1}}\right\} .
\end{aligned}
$$

Formally, for $\gamma_{G}<0$, Eq. (40) is related to the integral representation of the associated Legendre function (defined with a branch cut from $-\infty$ to 1) $Q_{\nu}^{\mu}(z)$, see Ref. 28. Since $n(k)$ is regular in $\gamma_{G}$ we can use analytic continuation to obtain for all non-integer $\gamma_{G}$

$$
\begin{aligned}
n(\tilde{k} ; T)= & \frac{2^{\gamma_{G}-1 / 2}}{\pi^{3 / 2}} \Gamma\left(-\gamma_{G}\right)\left(\frac{a}{\lambda_{T}}\right)^{2 \gamma_{G}} \\
\times & \operatorname{Re}\left\{e^{-i \pi \gamma_{G}}\left[\sinh \left(2 i a / \lambda_{T}\right)\right]^{-\left(\gamma_{G}+1 / 2\right)}\right. \\
& \times\left[e^{-i a / \lambda_{T}} Q_{-i \tilde{k} / 2-1}^{\gamma_{G}+1 / 2}\left[\cos \left(2 a / \lambda_{T}\right)\right]\right. \\
& \left.\left.-e^{i a / \lambda_{T}} Q_{-i \tilde{k} / 2}^{\gamma_{G}+1 / 2}\left[\cos \left(2 a / \lambda_{T}\right)\right]\right]\right\} .
\end{aligned}
$$

It can be shown that for $\gamma_{G} \lesssim 1 / 2$ and $k \lesssim\left(\lambda_{T} a\right)^{-1 / 2}$ this expression reduces, in the limit $a \rightarrow 0$, to

$$
\begin{aligned}
n(\tilde{k} ; T) & =\frac{\Gamma\left(-\gamma_{G}\right)}{2 \pi^{3 / 2}} \operatorname{Re}\left\{\Gamma\left(\gamma_{G}+1 / 2\right) e^{-i \pi\left(\gamma_{G}+1 / 2\right)}\right. \\
& \left.+i\left(\frac{a}{\lambda_{T}}\right)^{2 \gamma_{G}} \frac{\Gamma\left(1 / 2-\gamma_{G}\right) \Gamma\left[\left(1+2 \gamma_{G}-i \tilde{k}\right) / 2\right]}{\Gamma\left[\left(1-2 \gamma_{G}-i \tilde{k}\right) / 2\right]}\right\} .
\end{aligned}
$$

Using similar manipulations we obtain, for non-integer $\beta_{\mathcal{S}}=\beta_{c}+\beta_{s}$, the spin structure factor

$$
\begin{aligned}
S(\tilde{k} ; T) & =\int_{-\infty}^{\infty} d x e^{i\left(k+2 k_{F}\right) x} \tilde{S}(x, 0)=\int_{-\infty}^{\infty} \frac{d \omega}{2 \pi} S(k, \omega) \\
& =\frac{2^{\beta_{\mathcal{S}}-3 / 2}}{\pi^{5 / 2} a} \Gamma\left(1-\beta_{\mathcal{S}}\right)\left(\frac{a}{\lambda_{T}}\right)^{2 \beta_{\mathcal{S}}-1}
\end{aligned}
$$

$$
\begin{gathered}
\times \operatorname{Re}\left\{e^{i \pi\left(1 / 2-\beta_{\mathcal{S}}\right)}\left[\sinh \left(2 i a / \lambda_{T}\right)\right]^{1 / 2-\beta_{\mathcal{S}}}\right. \\
\left.\times Q_{-i \tilde{k} / 2-1 / 2}^{\beta_{\mathcal{S}}-1 / 2}\left[\cos \left(2 a / \lambda_{T}\right)\right]\right\},
\end{gathered}
$$

which for $a \rightarrow 0, \beta_{\mathcal{S}} \lesssim 3 / 2$ and $k \lesssim\left(\lambda_{T} a\right)^{-1 / 2}$ tends to

$$
\begin{aligned}
S(\tilde{k} ; T) & =\frac{\Gamma\left(1-\beta_{\mathcal{S}}\right)}{4 \pi^{3 / 2} a} \operatorname{Re}\left\{\Gamma\left(\beta_{\mathcal{S}}-1 / 2\right) e^{i \pi\left(1 / 2-\beta_{\mathcal{S}}\right)}\right. \\
& \left.+\left(\frac{a}{\lambda_{T}}\right)^{2 \beta_{\mathcal{S}}-1} \frac{\Gamma\left(1 / 2-\beta_{\mathcal{S}}\right) \Gamma\left(\beta_{\mathcal{S}}-i \tilde{k} / 2\right)}{\Gamma\left(1-\beta_{\mathcal{S}}-i \tilde{k} / 2\right)}\right\} .
\end{aligned}
$$

\section{THE LUTHER-EMERY LIQUID}

\section{A. The model}

In the Tomonaga-Luttinger liquid both the charge and spin excitations are gapless. Spin and charge gaps may open up as a result of adding to the Tomonaga-Luttinger Hamiltonian (II) terms describing backward and Umklapp scattering respectively. In the following we will assume that the 1DEG is sufficiently incommensurate so that Umklapp scattering may be neglected. Consequently the charge sector remains gapless and continues to be described by a free bosonic theory [the charge part of Eq. (8)]. Including a backward scattering term $g_{1} \sum_{\eta} \psi_{\eta, 1}^{\dagger} \psi_{-\eta,-1}^{\dagger} \psi_{\eta,-1} \psi_{-\eta, 1}$ results, after bosonization, in a spin Hamiltonian density of the sine-Gordon type

$$
\mathcal{H}_{s}=\frac{v_{s}}{2}\left[K_{s}\left(\partial_{x} \theta_{s}\right)^{2}+\frac{\left(\partial_{x} \phi_{s}\right)^{2}}{K_{s}}\right]+\frac{2 g_{1}}{(2 \pi a)^{2}} \cos \left(\sqrt{8 \pi} \phi_{s}\right) \text {. }
$$

The $g_{1}$ perturbation is relevant for $K_{s}<1$, in which case a spin gap js-dynamically generated according to the scaling relation $29 \Delta_{s} \sim\left(v_{s} / a\right)\left[g_{1} / 2 \pi^{2} v_{s}\right]^{1 /\left(2-2 K_{s}\right)}$ and the excitations are massive spin solitons. In the spin gapped phase the problem is most simply treated in terms of spin fermion fields and their mode decomposition $C_{ \pm}(\theta)$ (obeying the usual fermionic anti-commutation relations) in momentum space

$$
\begin{aligned}
\Psi_{\eta} & =F_{\eta} \exp \left[-i \sqrt{\pi / 2}\left(\theta_{s}-2 \eta \phi_{s}\right)\right] \\
& =\sqrt{\frac{\Delta_{s}}{4 \pi v_{s}}} \int_{-\infty}^{\infty} d \theta e^{\eta \theta / 2}\left[C_{+}(\theta) e^{i\left(x / \xi_{s}\right) \sinh \theta}-\eta C_{-}^{\dagger}(\theta) e^{-i\left(x / \xi_{s}\right) \sinh \theta}\right],
\end{aligned}
$$


where we introduce the spin correlation length $\xi_{s}=$ $v_{s} / \Delta_{s}$ and use the rapidity representation $k=\sinh \theta / \xi_{s}$.

For $K_{s}=1 / 2$, which is known as the free fermion or Luther-Emery point 20 , the refermionized Hamiltonian is non-interacting and massive with a gap $\Delta_{s}=g_{1} / 2 \pi a$

$$
\begin{aligned}
H_{s} & =\int d x \sum_{\eta= \pm 1}\left[-i v_{s} \eta \Psi_{\eta}^{\dagger} \partial_{x} \Psi_{\eta}+\Delta_{s} \Psi_{\eta}^{\dagger} \Psi_{-\eta}\right] \\
& =\int_{-\infty}^{\infty} d \theta E_{s}(\theta)\left[C_{+}^{\dagger}(\theta) C_{+}(\theta)+C_{-}^{\dagger}(\theta) C_{-}(\theta)-1\right],
\end{aligned}
$$

where the spin excitation spectrum is

$$
E_{s}(\theta) \equiv \Delta_{s} \cosh \theta=\sqrt{\Delta_{s}^{2}+\left(v_{s} k\right)^{2}} \equiv E_{s}(k) .
$$

In the following we will concentrate on computing the correlation functions at the Luther-Emery point. We will comment briefly on the effects of deviations from this point.

\section{B. The spin part of the spectral functions}

In contrast to the Tomonaga-Luttinger model the calculation of the spin part of most spectral functions for the Luther-Emery liquid is no longer trivial. The difficulty lies in the fact that generically the refermionized form for the spectral functions involves highly non-local operators. We start by evaluating the spin contribution to the transverse spin form factor, which fortunately has a simple representation in terms of the pseudo-fermions. We then consider the single hole spectral function that belongs to the wider class of functions which do not admit such a simple form. Interestingly, it is still possible to obtain an exact expression for this function too at the Luther-Emery point. This exact result extends and corrects earlier work of Voit21 and Wiegmann22.

In computing the various spectral properties of the system we can distinguish between two temperature regions. At temperatures large compared to $\Delta_{s}$ the spin gap can be ignored, and the results for the Tomonaga-Luttinger liquid apply. If the temperature is small compared to the spin gap we can evaluate the spin contribution to the correlation functions in the zero temperature limit while introducing errors of order $\exp \left(-\Delta_{s} / T\right)$. Henceforth we will be concerned with temperatures in the second region only.

\section{The spin part of $\mathcal{S}(k, \omega)$}

The spin piece of the transverse spin correlation function has the following simple form in terms of the spin fermion fields

$$
\tilde{\mathcal{S}}_{s}(x, t)=\left\langle\Psi_{1}^{\dagger}(x, t) \Psi_{-1}^{\dagger}(x, t) \Psi_{-1}(0,0) \Psi_{1}(0,0)\right\rangle .
$$

Since the theory reduces, at the Luther-Emery point, to a theory of free massive fermions, the corresponding spectral function can be readily computed with the result, for $T=0$,

$$
\mathcal{S}_{s}(k, \omega)=\frac{\omega^{2}-4 E_{s}^{2}(k / 2)}{4 v_{s}^{2}\left|q_{1} E_{s}\left(q_{2}\right)-q_{2} E_{s}\left(q_{1}\right)\right|} \Theta\left[-\omega-2 E_{s}(k / 2)\right],
$$

where the spin excitation spectrum $E_{s}(k)$ is given by Eq.(48) and $q_{1,2}$ are the solutions to the quadratic equation $\omega+E_{s}(q)+E_{s}(k-q)=0$, that is

$$
q_{1,2}=\frac{k}{2} \pm \frac{\omega}{2 v_{s}} \sqrt{1+\frac{4 \Delta_{s}^{2}}{v_{s}^{2} k^{2}-\omega^{2}}} .
$$

\section{The spin part of $G^{<}(k, \omega)$}

The refermionized form of the single hole Green function (below we consider the case $\eta=-1$ and $\sigma=1$ )

$$
\tilde{G}_{s}(x, t)=\left\langle U_{\frac{1}{4}}^{\dagger}(x, t) \Psi_{-1}^{\dagger}(x, t) \Psi_{-1}(0,0) U_{\frac{1}{4}}(0,0)\right\rangle,
$$

involves the non-local vertex operators

$$
U_{\alpha}(x)=\exp \left[i \sqrt{8 \pi} \alpha \phi_{s}(x)\right],
$$

with

$$
\phi_{s}(x)=\sqrt{\frac{\pi}{2}} \sum_{\eta= \pm 1} \int^{x} d y \Psi_{\eta}^{\dagger}(y) \Psi_{\eta}(y) .
$$

From Eqs. (46), (53) and (54) it is evident that $\Psi_{\eta}$ and $U_{\alpha}$ create and destroy a single spin soliton or an integer number of soliton pairs, respectively. Therefor, the Green function consists of a coherent one spin soliton piece and an incoherent multi-soliton piece 30

$$
G_{s}(k, \omega)=Z_{s}(k) \delta\left[\omega+E_{s}(k)\right]+G_{s}^{(m u l t i)}(k, \omega),
$$

where the multi-soliton piece is proportional (at $T=0$ ) to $\Theta\left[-\omega-3 E_{s}(k / 3)\right]$. Deviations from the Luther-Emery point in the case $K_{s}<1 / 2$ will result in the formation of a spin soliton-antisoliton bound state, a "breather", which can shift the threshold energy for multi-soliton excitations somewhat.

At the Luther-Emery point the form factors of the vertex operators, i.e. their matrix elements between the vacuum and various multi-soliton states, are known exactly. This fact enables us to obtain exact results for the different parts of the spectral function. The details of the calculation are presented in Appendix B. For the spectral weight of the coherent piece we find

$$
Z_{s}(k)=\frac{8 c^{2}}{\pi}\left(\frac{2 \xi_{s}}{a}\right)^{\frac{3}{8}}\left[1-\frac{v_{s} k}{E_{s}(k)}\right],
$$


where $c=0.101$. We would like to note here that a simple scaling argument 30 allows us to obtain the dependence of $Z_{s}$ on $\xi_{s}$ for arbitrary $K_{s}<1$. It follows from the observation that the sine-Gordon theory is asymptotically free and hence the dependence of $G_{s}$ on the short distance cutoff $a$ is unaffected by the opening of a spin gap. Since in the absence of a gap $G_{s}$ is proportional to $a^{2 \gamma_{s}-1 / 2}$ it is a matter of dimensional analysis to see that

$$
Z_{s}(k)=\left(\xi_{s} / a\right)^{\frac{1}{2}-2 \gamma_{s}} f_{s}\left(k \xi_{s}\right),
$$

where $f_{s}$ is an undetermined scaling function.

The incoherent piece of $G_{s}^{<}(k, \omega)$ consists of contributions from processes involving intermediate states containing $N=2 n+1$ spin solitons, $n=1,2,3, \cdots$. Each such contribution starts at an energy threshold $-N E_{s}(k / N)$

$$
G_{s}^{(m u l t i)}(k, \omega)=\sum_{N=3,5, \cdots} G_{s}^{(N \mathrm{sol})}(k, \omega) \Theta\left[-\omega-N E_{s}\left(\frac{k}{N}\right)\right] .
$$

At the vicinity of the threshold i.e. for $k \xi_{s} \ll 1$ and $\left|\omega+N E_{s}(k / N)\right| / \Delta_{s} \ll 1$ the behavior of $G_{s}^{(N \text { sol })}$ is given by $\left[\omega+N E_{s}(k / N)\right]^{n^{2}+n-1}$. In particular the 3 soliton part reads then

$G_{s}^{(3 \mathrm{sol})}(k, \omega)=\frac{8 c^{2}}{\sqrt{3} \pi^{2}}\left(\frac{2 \xi_{s}}{a}\right)^{\frac{3}{8}}\left[\frac{-\omega-3 E_{s}(k / 3)}{\Delta_{s}^{2}}\right]$.

As discussed above, for large $|\omega|$ the incoherent piece should asymptotically approach the Tomonaga-Luttinger result $\omega^{2 \gamma_{s}-3 / 2}$.

\section{The single hole spectral function}

Once the spin part is calculated there still remains the task of convolving it with the corresponding charge part in order to obtain an expression for the full spectral function. Analytically this is difficult, and like in the case of the Tomonaga-Luttinger liquid, progress can be made only in special cases. Below we carry out the convolution for the single hole spectral function. We consider temperatures well below the spin gap scale and correspondingly use the above derived zero temperature results for the spin part. For the gapless charge degrees of freedom we continue to use the finite temperature TomonagaLuttinger expressions.

The spectral function can be written as a sum of two contributions $G^{<}=G_{1}+G_{2}$ coming from the convolution of the charge part with the coherent (single soliton) and incoherent (multi-soliton) pieces of $G_{s}^{<}$. The frequency integral in $G_{1}$ is readily evaluated with the result

$$
G_{1}(k, \omega ; T)=\frac{c^{2}}{\pi^{3}} \frac{\lambda_{T}^{2}}{v_{c}}\left(\frac{a}{\lambda_{T}}\right)^{2 \gamma_{c}+\frac{1}{2}}\left(\frac{2 \xi_{s}}{a}\right)^{\frac{3}{8}}
$$

$$
\begin{aligned}
& \times \int d q\left[1-\frac{v_{s}(k-q)}{E_{s}(k-q)}\right] h_{\gamma_{c}+\frac{1}{2}}\left[\frac{\omega+E_{s}(k-q)+v_{c} q}{2 \pi T}\right] \\
& \times h_{\gamma_{c}}\left[\frac{\omega+E_{s}(k-q)-v_{c} q}{2 \pi T}\right],
\end{aligned}
$$

where here $\lambda_{T}=v_{c} / \pi T$.

In Fig. 2 we present representative contributions of the single spin soliton piece, $G_{1}$, to the MDCs and EDCs of a Luther-Emery liquid with various values of the charge exponent $\gamma_{c}$. Using some of the results derived below, we also indicate the asymptotic behavior of the three spin soliton contribution, $G_{2}^{(3 \mathrm{sol})}$, to the EDCs in the vicinity of its zero temperature threshold $\omega=-3 \Delta_{s}$.
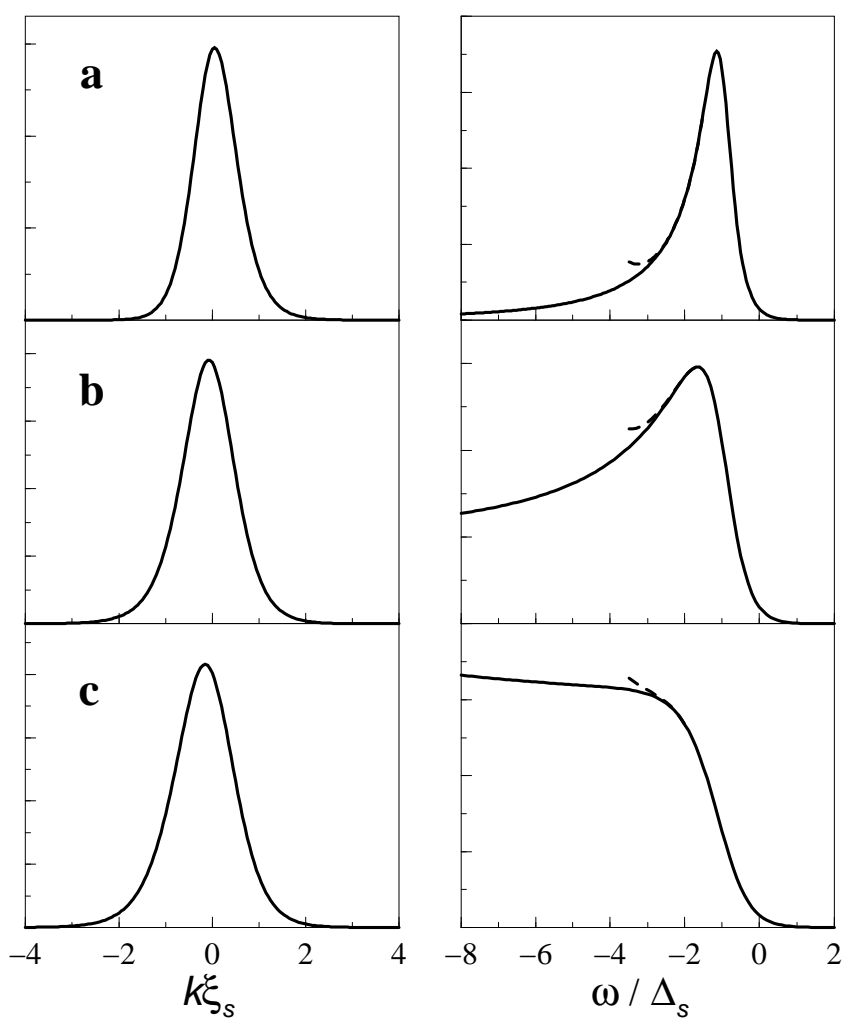

FIG. 2. The single spin soliton contribution, $G_{1}$, to the MDCs at $\omega=0$ (left) and EDCs at $k=0$ (right), of a Luther-Emery liquid $\left(K_{s}=1 / 2\right)$, with $v_{c} / v_{s}=3, \Delta_{s} / T=3$ and a) $\gamma_{c}=0$, b) $\gamma_{c}=0.2$, and c) $\gamma_{c}=0.4$. The asymptotic contribution of the three spin soliton piece, $G_{2}^{(3 \text { sol })}$, to the EDCs, near its zero temperature threshold $\omega=-3 \Delta_{s}$, is indicated by the dashed lines. This contribution was calculated using Eq. (65) in the case $\gamma_{c}=0$. For the EDCs with $\gamma_{c}=0.2,0.4$ we used the asymptotic result for the case $v_{s} / v_{c} \rightarrow 0$, Eq. 63). Other multi-soliton processes start to contribute near $\omega / \Delta_{s}=5,7, \cdots$, as discussed in the text.

We now restrict the discussion to the following special circumstances: 


\section{The case $v_{s} / v_{c} \rightarrow 0$}

In this case the remaining integral in (60) is straightforward since the $q$ dependence of the spin part and $E_{s}(k-q)$ disappears and we are left with

$$
\begin{aligned}
G_{1}(k, \omega ; T) & =\frac{4 c^{2}}{\pi^{2}} \frac{a}{v_{c}}\left(\frac{a}{\lambda_{T}}\right)^{2 \gamma_{c}-\frac{1}{2}}\left(\frac{2 \xi_{s}}{a}\right)^{\frac{3}{8}} \\
& \times\left[1-\frac{v_{s} k}{E_{s}(k)}\right] h_{2 \gamma_{c}+\frac{1}{2}}\left[\frac{\omega+E_{s}(k)}{\pi T}\right],
\end{aligned}
$$

which in the limit of zero temperature reduces to

$$
\begin{aligned}
G_{1}(k, \omega ; 0) & =\frac{8 c^{2}}{\pi} \frac{1}{\Gamma\left(2 \gamma_{c}+1 / 2\right)}\left(\frac{a}{v_{c}}\right)^{2 \gamma_{c}+\frac{1}{2}}\left(\frac{2 \xi_{s}}{a}\right)^{\frac{3}{8}} \\
& \times\left[-\omega-E_{s}(k)\right]^{2 \gamma_{c}-\frac{1}{2}} \Theta\left[-\omega-E_{s}(k)\right] .
\end{aligned}
$$

For the 3 -soliton contribution to $G_{2}$ we obtain, assuming $k \xi_{s} \ll 1$ and $\left|\omega+3 E_{s}(k / 3)\right| \ll \Delta_{s}$,

$$
\begin{aligned}
& G_{2}^{(3 \mathrm{sol})}(k, \omega ; T)=\frac{4 c^{2}}{\sqrt{3} \pi^{4}} \frac{1}{\Delta_{s} T}\left(\frac{a}{\lambda_{T}}\right)^{2 \gamma_{c}+\frac{1}{2}}\left(\frac{2 \xi_{s}}{a}\right)^{\frac{3}{8}} \\
& \times \int_{\omega+3 E_{s}(k / 3)}^{\infty} d \nu h_{2 \gamma_{c}+\frac{1}{2}}\left(\frac{\nu}{\pi T}\right)\left[\frac{\nu-\omega-3 E_{s}(k / 3)}{\Delta_{s}}\right],
\end{aligned}
$$

which at $T=0$ equals

$$
\begin{array}{r}
G_{2}^{(3 \mathrm{sol})}(k, \omega ; 0)=\frac{8 c^{2}}{\sqrt{3} \pi^{2}} \frac{1}{\Gamma\left(2 \gamma_{c}+5 / 2\right)}\left(\frac{a}{v_{c}}\right)^{2 \gamma_{c}+\frac{1}{2}}\left(\frac{2 \xi_{s}}{a}\right)^{\frac{3}{8}} \\
\times \frac{1}{\Delta_{s}^{2}}\left[-\omega-3 E_{s}(k / 3)\right]^{2 \gamma_{c}+\frac{3}{2}} \Theta\left[-\omega-3 E_{s}(k / 3)\right] .
\end{array}
$$

For this case the $N=2 n+1$ soliton contribution to $G_{2}$ is proportional, in the vicinity of its threshold, to $\left[-\omega-N E_{s}(k / N)\right]^{2 \gamma_{c}+n^{2}+n-1 / 2} \Theta\left[-\omega-N E_{s}(k / N)\right]$.
2. the case $\gamma_{c}=0$

It is also possible to derive closed expressions for the spectral function when $\gamma_{c}=0$ but $r=v_{s} / v_{c}$ is arbitrary.

$$
\begin{aligned}
G_{1}(k, \omega & ; T)=\frac{4 c^{2}}{\pi^{2}} \frac{1}{1-r} \frac{\sqrt{a \lambda_{T}}}{v_{c}}\left(\frac{2 \xi_{s}}{a}\right)^{\frac{3}{8}} \\
\times & h_{\frac{1}{2}}\left[\frac{\omega-r v_{s} k+\sqrt{\left(r \omega-v_{s} k\right)^{2}+\left(1-r^{2}\right) \Delta_{s}^{2}}}{\pi\left(1-r^{2}\right) T}\right] \\
& \times \frac{r \omega-v_{s} k+\sqrt{\left(r \omega-v_{s} k\right)^{2}+\left(1-r^{2}\right) \Delta_{s}^{2}}}{\sqrt{\left(r \omega-v_{s} k\right)^{2}+\left(1-r^{2}\right)_{s}^{2}}} .
\end{aligned}
$$

Using the asymptotic form (A9) of $h_{\gamma}(k)$ one can obtain the zero temperature limit of $G_{1}$. When $r<1$ we find

$$
\begin{aligned}
G_{1}(k, \omega & ; 0)=\frac{8 c^{2}}{\pi^{3 / 2}}\left(\frac{1+r}{1-r}\right)^{\frac{1}{2}}\left(\frac{a}{v_{c}}\right)^{\frac{1}{2}}\left(\frac{2 \xi_{s}}{a}\right)^{\frac{3}{8}} \\
& \times \frac{r \omega-v_{s} k+\sqrt{\left(r \omega-v_{s} k\right)^{2}+\left(1-r^{2}\right) \Delta_{s}^{2}}}{\sqrt{\left(r \omega-v_{s} k\right)^{2}+\left(1-r^{2}\right) \Delta_{s}^{2}}} \\
& \times \frac{\Theta\left[-\omega-E_{s}(k)\right]}{\sqrt{-\omega+r v_{s} k-\sqrt{\left(r \omega-v_{s} k\right)^{2}+\left(1-r^{2}\right) \Delta_{s}^{2}}}} .
\end{aligned}
$$

The kinematics in the case $r>1$ is more involved. In particular we find that for sufficiently large $r$ it is possible to distribute the total momentum between the spin soliton and the gapless charge modes in a way that gives a contribution at frequencies smaller than $E_{s}(k)$. (In this case the effect exists only for $k<0$. This is a special property of the point $\gamma_{c}=0$ which precludes mixing of left and right moving charge excitations.)

$$
\begin{aligned}
G_{1}(k, \omega ; 0)= & \frac{8 c^{2}}{\pi^{3 / 2}}\left(\frac{r+1}{r-1}\right)^{\frac{1}{2}}\left(\frac{a}{v_{c}}\right)^{\frac{1}{2}}\left(\frac{2 \xi_{s}}{a}\right)^{\frac{3}{8}} \\
\times & \sum_{\sigma} \frac{v_{s} k-r \omega-\sigma \sqrt{\left(r \omega-v_{s} k\right)^{2}+\left(1-r^{2}\right) \Delta_{s}^{2}}}{\sqrt{\left(r \omega-v_{s} k\right)^{2}+\left(1-r^{2}\right) \Delta_{s}^{2}} \sqrt{\omega-r v_{s} k+\sigma \sqrt{\left(r \omega-v_{s} k\right)^{2}+\left(1-r^{2}\right) \Delta_{s}^{2}}}} \\
& \left\{\begin{array}{l}
\sigma=1 \quad \text { if } \omega<-E_{s}(k) \\
\sigma=-1,1 \text { if } k \xi_{s}<-\frac{1}{\sqrt{r^{2}-1}} \text { and }-E_{s}(k)<\omega<\frac{v_{s} k-\sqrt{r^{2}-1} \Delta_{s}}{r}
\end{array}\right.
\end{aligned}
$$

For $k \xi_{s} \ll 1$ and $\left|\omega+3 E_{s}(k / 3)\right| \ll \Delta_{s}$ the 3 -soliton contribution to $G_{2}$ reads

$$
G_{2}^{(3 \mathrm{sol})}(k, \omega ; T)=\frac{4 c^{2}}{\sqrt{3} \pi^{3}} \frac{\sqrt{a \lambda_{T}}}{\Delta_{s}}\left(\frac{2 \xi_{s}}{a}\right)^{\frac{3}{8}} \int d q h_{\frac{1}{2}}\left(\lambda_{T} q\right)
$$

$$
\begin{aligned}
& \times\left[\frac{v_{c} q-\omega-3 E_{s}[(k-q) / 3]}{\Delta_{s}}\right] \\
& \times \Theta\left\{v_{c} q-\omega-3 E_{s}[(k-q) / 3]\right\} .
\end{aligned}
$$

When $r<1$ the zero temperature limit of the above is readily evaluated giving 


$$
\begin{aligned}
G_{2}^{(3 \mathrm{sol})}(k, \omega ; 0)=\frac{32 c^{2}}{3^{3 / 2} \pi^{5 / 2}}\left(\frac{a}{\Delta_{s} v_{c}}\right)^{\frac{1}{2}}\left(\frac{2 \xi_{s}}{a}\right)^{\frac{3}{8}} \\
\quad \times\left[-\frac{\omega+3 E_{s}(k / 3)}{\Delta_{s}}\right]^{\frac{3}{2}} \Theta\left[-\omega-3 E_{s}(k / 3)\right] .
\end{aligned}
$$

The zero temperature limit when $r>1$ is, once again, more complicated. However, it is possible to show that in the range of validity of Eq. (68), i.e. for $k \xi_{s} \ll 1$, and for $1<r \lesssim 3$ it coincides with the expression for the case $r<1 \mathrm{Eq}$. (69). Deviations from this behavior may occur only if the velocity ratio is large and satisfies $r \gg 3$.

\section{ACKNOWLEDGMENTS}

It is a pleasure to thank S. Kivelson for many useful discussions and comments.

\section{APPENDIX A: THE FUNCTION $h_{\gamma}(k)$}

The function $h_{\gamma}(k)$ is real

$$
\begin{aligned}
h_{\gamma}(k) & =\int_{-\infty}^{\infty} d x e^{i k x} h_{\gamma}(x) \\
& =\lim _{a \rightarrow 0} 2 \operatorname{Re}\left\{\int_{0}^{\infty} d x \frac{e^{i k x}}{[-i \sinh (x+i a)]^{\gamma}}\right\} .
\end{aligned}
$$

Although the imaginary part of the integral diverges as $a^{1-\gamma}$ for $\gamma>1$, its real part, and hence also $h_{\gamma}(k)$, are analytic for all values of $\gamma$. Substituting $y=e^{-2 x}$ we find

$$
h_{\gamma}(k)=\lim _{a \rightarrow 0} \operatorname{Re}\left\{(2 i)^{\gamma} \int_{0}^{1} d y y^{\frac{\gamma-i k}{2}-1}\left(1-e^{-2 i a} y\right)^{-\gamma}\right\} \text {. }
$$

The integral is analytic in the limit $a \rightarrow 0$ for $\gamma<1$. In this range the exponential factor in (A2) can be dropped and it reduces to the integral representation of the beta function 31 . We can then use analytical continuation to obtain for all non-integer values of $\gamma$

$$
h_{\gamma}(k)=\operatorname{Re}\left[(2 i)^{\gamma} B\left(\frac{\gamma-i k}{2}, 1-\gamma\right)\right] .
$$

For the integers, $h_{n}(k)$ can be calculated as follows. First, we obtain using standard residue technique

$$
\begin{aligned}
& h_{1}(k)=2 \pi f_{+}(\pi k), \\
& h_{2}(k)=2 \pi k f_{-}(\pi k),
\end{aligned}
$$

where $f_{ \pm}(k)=\left(e^{k} \pm 1\right)^{-1}$ are the fermionic and bosonic occupation functions. We then integrate (A1) by parts twice to find the recursion relation

$$
h_{n+2}(k)=\frac{k^{2}+n^{2}}{n(n+1)} h_{n}(k),
$$

which implies

$$
\begin{aligned}
h_{2 n+1}(k) & =\frac{2 \pi}{\Gamma(2 n+1)} \prod_{m=0}^{n-1}\left[(1+2 m)^{2}+k^{2}\right] f_{+}(\pi k), \\
h_{2 n}(k) & =\frac{2 \pi}{\Gamma(2 n)} k \prod_{m=1}^{n-1}\left[(2 m)^{2}+k^{2}\right] f_{-}(\pi k) .
\end{aligned}
$$

We also note that

$$
h_{0}(k)=2 \pi \delta(k) .
$$

Finally, the asymptotic behavior of $h_{\gamma}(k)$ for large $|k|$ is easily evaluated with the result

$$
h_{\gamma}(|k| \rightarrow \infty)=\frac{2 \pi}{\Gamma(\gamma)} \Theta(-k)(-k)^{\gamma-1} .
$$

Since the spectral functions have a scaling form in the variables $\omega / T$ and $v_{\alpha} k / T$ Eq. (A9) also determines the zero temperature limit of these functions.

\section{APPENDIX B: CALCULATING $G_{s}^{<}(k, \omega)$ AT THE LUTHER-EMERY POINT}

Inserting the resolution of the identity into Eq. (52) one obtains

$$
\begin{aligned}
\tilde{G}_{s}^{<}(x, t)=\sum_{N, N^{\prime}}\left\langle 0\left|U_{\frac{1}{4}}^{\dagger}(x, t)\right| N\right\rangle & \left\langle N\left|\Psi_{-1}^{\dagger}(x, t) \Psi_{-1}(0,0)\right| N^{\prime}\right\rangle \\
& \times\left\langle N^{\prime}\left|U_{\frac{1}{4}}(0,0)\right| 0\right\rangle . \quad(\mathrm{B} 1)
\end{aligned}
$$

The matrix elements of the vertex operators appearing in (B1) are known as the form factors of these operators. They have been derived, at the Luther-Emery point (see also Ref. 32), by a variety of ways. They were first obtained by Schroer and Truong 33 who normal ordered the vertex operator with respect to the spin fermions. Smirnov 34 derived them for $\alpha=1 / 2$ using bootstrap axioms. Most recently they were calculatef using monodromy relations by Bernard and LeClair 35 . Since the fields $U_{\alpha}(0,0)$ are neutral with respect to the topological $U(1)$ charge of the solitons $( \pm)$ the form factors are non-vanishing only for $U(1)$ neutral states

$$
\left\langle 0\left|U_{\alpha}(x, t) C_{+}^{\dagger}\left(\theta_{2 n}\right) \cdots C_{+}^{\dagger}\left(\theta_{n+1}\right) C_{-}^{\dagger}\left(\theta_{n}\right) \cdots C_{-}^{\dagger}\left(\theta_{1}\right)\right| 0\right\rangle=V_{\alpha}(-1)^{n(n-1) / 2}\left(\frac{\sin \pi \alpha}{2 \pi i}\right)^{n}
$$




$$
\times \exp \left[i \sum_{k=1}^{2 n}\left(\frac{x}{\xi_{s}} \sinh \theta_{k}-\Delta_{s} t \cosh \theta_{k}\right)+\alpha \sum_{k=1}^{n}\left(\theta_{n+k}-\theta_{k}\right)\right] \frac{\prod_{1 \leq k<j \leq n} \sinh \left(\frac{\theta_{k}-\theta_{j}}{2}\right) \sinh \left(\frac{\theta_{n+k}-\theta_{n+j}}{2}\right)}{\prod_{1 \leq k, j \leq n} \cosh \left(\frac{\theta_{n+k}-\theta_{j}}{2}\right)}
$$

where $V_{\alpha}$, the vacuum expectation value of the vertex operators, is given by 3

$$
V_{\alpha} \equiv\left\langle 0\left|U_{\alpha}(0,0)\right| 0\right\rangle=c(\alpha)\left(\frac{2 \xi_{s}}{a}\right)^{-\alpha^{2}},
$$

with

$$
c(\alpha)=\exp \left\{\int_{0}^{\infty} \frac{d t}{t}\left[\frac{\sinh ^{2}(\alpha t)}{\sinh ^{2} t}-\alpha^{2} e^{-2 t}\right]\right\} .
$$

Furthermore, since $\Psi_{\eta}$ creates and destroys a single soliton the state $\left|N^{\prime}\right\rangle$ can differ from $|N\rangle$ by $0, \pm 2$ solitons only. It is also easy to check that $G_{s}^{<}(k, \omega)$ is real. Using Eqs. (46) and (B2) one obtains that each term in the Fourier transform of Eq. (B1) is proportional to $i^{\left(N+N^{\prime}\right) / 2}$ times a real expression. Thus only terms with $\left(N+N^{\prime}\right) / 2$ an even integer should be considered. Combining these two observations we conclude that the contribution to $G_{s}^{<}(k, \omega)$ comes solely from the terms $N=N^{\prime}$ (the amplitudes for the cases $N=N^{\prime} \pm 2$ are finite but cancel each other).

The coherent piece of the spectral function is due to the terms $N=0$ and $N=2$. The evaluation of the first is straightforward with the result

$$
\pi V^{2}\left[1-\frac{v_{s} k}{E_{s}(k)}\right] \delta\left[\omega+E_{s}(k)\right],
$$

where $V=V_{\frac{1}{4}}=c\left(\frac{1}{4}\right)\left(2 \xi_{s} / a\right)^{-1 / 16}$. The cutoff dependence of the above result is in conflict with the general scaling argument that was given following Eq. (56). It represents corrections to scaling coming from irrelevant operators. The leading scaling behavior is recovered by considering the contribution of the $N=2$ term to the coherent piece

$$
\frac{V^{2}}{32 \pi^{3} \xi_{s}}\left[\int d \beta \frac{e^{-\frac{3}{4} \beta}}{\cosh \frac{\beta}{2}}\right]^{2} \int d \theta e^{-\theta+i\left[\left(x / \xi_{s}\right) \sinh \theta-\Delta_{s} t \cosh \theta\right]} .
$$

The $\beta$ integral is divergent and a lower cutoff $-\ln \left(2 \xi_{s} / a\right)$ (corresponding to a cutoff $k=-1 / a$ ) should be introduced. We then find for the leading contribution to the coherent piece

$$
\begin{aligned}
\tilde{G}_{s}^{(\text {coher })}(x, t)= & \frac{4 c^{2}}{i \pi^{3} \xi_{s}}\left(\frac{2 \xi_{s}}{a}\right)^{\frac{3}{8}} \frac{x-v_{s} t}{\sqrt{x^{2}-\left(v_{s} t\right)^{2}+i \epsilon t}} \\
& \times K_{1}\left[\frac{\sqrt{x^{2}-\left(v_{s} t\right)^{2}+i \epsilon t}}{\xi_{s}}\right], \quad
\end{aligned}
$$

where $K_{1}(x)$ is a modified Bessel function. Fourier transforming it one obtains $Z_{s}(k) \delta\left[\omega+E_{s}(k)\right]$ with the spectral weight $Z_{s}(k)$ given by Eq. (56).

The $2 n+1$ soliton contribution to the incoherent piece of the spectral function comes from the terms $N=2 n$ and $N=2 n+2$. As is the case with the coherent piece the former contains corrections to scaling while the latter is responsible for the leading behavior which is proportional to

$$
\begin{aligned}
V^{2} \int d \theta_{1} \cdots d \theta_{2 n+1} & {\left[\int d \beta e^{-\frac{3}{4} \beta} \frac{\prod_{n+2 \leq j \leq 2 n+1} \sinh \left(\frac{\theta_{j}-\beta}{2}\right)}{\prod_{1 \leq j \leq n+1} \cosh \left(\frac{\theta_{j}-\beta}{2}\right)}\right]^{2} \delta\left(k \xi_{s}-\sum_{i=1}^{2 n+1} \sinh \theta_{i}\right) \delta\left(\frac{\omega}{\Delta_{s}}+\sum_{i=1}^{2 n+1} \cosh \theta_{i}\right) } \\
& \times \exp \left(\frac{1}{2} \sum_{j=1}^{n+1} \theta_{j}-\frac{1}{2} \sum_{j=n+2}^{2 n+1} \theta_{j}\right) \frac{\prod_{1 \leq k<j \leq n+1} \sinh ^{2}\left(\frac{\theta_{k}-\theta_{j}}{2}\right) \prod_{n+2 \leq k<j \leq 2 n+1} \sinh ^{2}\left(\frac{\theta_{k}-\theta_{j}}{2}\right)}{\prod_{n+2 \leq k \leq 2 n+1,1 \leq j \leq n+1} \cosh ^{2}\left(\frac{\theta_{k}-\theta_{j}}{2}\right)} .
\end{aligned}
$$

The exact evaluation of the above integrals is difficult but their behavior near the threshold $(2 n+1) E_{s}[k /(2 n+1)]$ may be extracted in the following way. Writing $\omega=$ $-(2 n+1) E_{s}[k /(2 n+1)]-\Delta \omega$ one finds that for $k \xi_{s} \ll 1$ and $\Delta \omega \ll \Delta_{s}$ the second $\delta$-function restricts the integration region over $\theta_{i}$ to a small ball near the origin. It is then legitimate to expand the integrand to lowest order in these variables. The integral over $\beta$ gives (together with the factor $V^{2}$ ) the overall cutoff dependence $\left(2 \xi_{s} / a\right)^{3 / 8}$ in accord with the general scaling argument.
By changing variables to spherical coordinates it is easy to check that the remaining integral over $\theta_{i}$ is proportional to $\Delta \omega^{n^{2}+n-1} \Theta(\Delta \omega)$. A detailed evaluation of the 3 solitons case results in Eq. (59). 
${ }^{1}$ S. Tomonaga, prog. Theor. Phys. 5, 544 (1950).

${ }^{2}$ K. D. Schotte and U. Schotte, Phys. Rev. 182, 479 (1969).

${ }^{3}$ D. C. Mattis, J. Math. Phys. 15, 609 (1974).

${ }^{4}$ A. Luther and I. Peschel, Phys. Rev. B 9, 2911 (1974).

${ }^{5}$ For a review, see A. J. Heeger, S. A. Kivelson, J. R. Schrieffer, and W. P. Su, Rev. Mod. Phys. 60, 781 (1988).

${ }^{6}$ For a review, see C. Bourbonnais and D. Jerome in $A d$ vances in Synthetic Metals, Twenty Years of Progress in Science and Technology, edited by P. Bernier, S. Lefrant, and G. Bidan (Elsevier, New York, 1999), p. 206.

${ }^{7}$ P. Monceau, Electronic Properties of Inorganic Quasi-OneDimensional Compounds, (D. Reidel Publ. Co., Dordrecht, Boston, 1985).

${ }^{8}$ G. Gruner, Density Waves in Solids, (Addison-Wesley, 1994).

${ }^{9}$ X. G. Wen, Phys. Rev. B 41, 12838 (1990); F. P. Milliken, C. P. Umbach, and R. A. Webb, Sol. State Comm. 97, 309 (1996); A. M. Chang, L. N. Pfeiffer and K. W. West, Phys. Rev. Lett. 77, 2538 (1996); M. Grayson, D. C. Tsui, L. N. Pfeiffer, K. W. West, and A. M. Chang, ibid. 80, 1062 (1998).

${ }^{10}$ S. Tarucha, T. Honda, and T. Saku, Solid State Comm. 94, 413 (1995); A. Yacoby, H. L. Stormer, N. S. Wingreen, K. W. Baldwin, and K. W. West, Phys. Rev. Lett. 77, 4612 (1996).

${ }^{11}$ For a recent perspective on stripe phases in high temperature superconductors see, V. J. Emery, S. A. Kivelson, and J. M. Tranquada, Proc. Natl. Acad. Sci. USA 96, 8814 (1999).

${ }^{12}$ F. Zwick, S. Brown, G. Margaritondo, C. Merlic, M. Onellion, J. Voit, and M. Grioni, Phys. Rev. Lett. 79, 3982 (1997).

${ }^{13}$ Y. Hwu, P. Almares, M. Marsi, H. Berger, F. Lévy, M. Grioni, D. Malterre, and G. Margaritondo, Phys. Rev. B 46, 13624 (1992); A. Terassi, M. Marsi, H. Berger, G. Margaritondo, R. J. Kelly, and M. Onellion, ibid. 52, 5592 (1995).

14 M. Grioni, H. Berger, M. Garnier, F. Bommeli, L. Degiorgi, and S. Schlenker, Physica Scripta T 66, 172 (1996).

${ }^{15}$ X. J. Zhou, P. Bogdanov, S. A. Kellar, T. Noda, H. Eisaki, S. Uchida, Z. Hussain, and Z.-X. Shen, Science 286, 268 (1999).
${ }^{16}$ V. Meden and K. Schönhammer, Phys. Rev. B 46, 15753 (1992).

17 J. Voit, Phys. Rev. B 47, 6740 (1993).

${ }^{18}$ K. Schönhammer and V. Meden, J. Electron Spectrosc. Relat. Phenom. 62, 225 (1993).

19 N. Nakamura and Y. Suzumura, Prog. Theor. Phys. 98, 29 (1997).

${ }^{20}$ A. Luther and V. J. Emery, Phys. Rev. Lett. 33, 589 (1974).

${ }^{21}$ J. Voit, Euro. Phys. J. B 5, 505 (1998).

${ }^{22}$ P. B. Wiegmann, Phys. Rev. B 59, 15705 (1999).

${ }^{23}$ V. J. Emery, in Highly Conducting One-Dimensional Solids, edited by J. T. Devreese, R. P. Evrard and V. E. van Doren (Plenum, New York 1979), p. 247.

24 J. von Delft and H. Schoeller, Annalen Phys. 7, 225 (1998).

${ }^{25}$ A. E. Mattsson, S. Eggert and H. Johannesson, Phys. Rev. B 56, 15615 (1997).

${ }^{26}$ S. Eggert, A. E. Mattsson and J. M. Kinaret, Phys. Rev. B 56, R15537 (1997).

${ }^{27}$ N. Kawakami and S. K. Yang, Phys. Lett. A 148, 359 (1990).

28 A. Erdèlyi et al., Higher Transcendental Functions (McGraw Hill, New York, 1953) Vol. I, p. 155.

${ }^{29}$ See, for example, A. O. Gogolin, A. A. Nersesyan and A. M. Tsvelik, Bosonization and Strongly Correlated Systems, ( Cambridge University Press, Cambridge, 1998).

${ }^{30}$ E. W. Carlson, D. Orgad, S. A. Kivelson and V. J. Emery, Phys. Rev B 62, 3422 (2000).

${ }^{31}$ I.S. Gradshteyn and I. M. Ryzhik, Table of Integrals, Series and Products (Academic, New York, 1994) p. 957.

32 An integral representation for the form factors away from the Luther-Emery point is suggested in S. Lukyanov, Phys. Lett. A 12, 2543 (1997).

${ }^{33}$ B. Schroer and T. T. Truong, Nucl. Phys. B 144, 80 (1978).

${ }^{34}$ F. A. Smirnov, Form factors in Completely Integrable Models of Quantum Field Theory, Advanced Series in Mathematical Physics 14 (World Scientific, 1992).

${ }^{35}$ D. Bernard and A. LeClair, Nucl. Phys. B 426, 534 (1994).

${ }^{36}$ S. Lukyanov and A. Zamolodchikov, Nucl. Phys. B 493, 571 (1997). 